\title{
Numerical Simulations of Morphology, Flow Structures and Forces for a Sonic Jet Exhausting in Supersonic Crossflow
}

\author{
S. B. H. Shah ${ }^{1 \dagger}$, S. Zahir ${ }^{2}$ and X. Y. Lu \\ ${ }^{1}$ Department of Modern Mechanics, University of Science and Technology of China, \\ ${ }^{2}$ National Engineering and Scientific Commission, Islamabad, Pakistan \\ †Corresponding Author Email: b_bukhari@yahoo.com
}

(Received December 26, 2009; accepted March 15, 2010)

\begin{abstract}
A numerical study is performed for a sonic jet issuing from a blunted cone to provide possible directional control in supersonic crossflow by solving the unsteady Reynolds-averaged Navier-Stokes (RANS) equations with the twoequation $k-\omega$ turbulence model. Results are presented in the form of static aerodynamic coefficients, computed at a free stream Mach number 4.0, with varying pressure ratios, incidence angle and keeping zero yaw and roll angles. The morphology and flow structure for the jet exhausting in crossflow at various pressure ratios is described in detail. The Flight control of the projectile can be accomplished by taking advantage of a complex shock-boundary layer interaction produced by jet interacting with the oncoming crossflow by altering pressure distribution in vicinity of the jet, a net increase in the net force can be utilized for maneuvering of vehicle and possible flight control. Computed static aerodynamic coefficients and pressure distribution using CFD analyses is with an accuracy of $\pm 5 \%$ in the supersonic range.
\end{abstract}

Keywords: Jet, Crossflow, Shock boundary layer interaction, Directional Control.

\section{NOMENCLATURE}

$\begin{array}{ll}A & \text { Reference Area } \\ C_{M} & \text { Pitching moment Coefficient, } M /\left(q_{\infty} A L\right) \\ C_{p} & \text { Pressure Coefficient, }\left(p-p_{\infty}\right) / q_{\infty} \\ C_{X} & \text { Axial Force Coefficient, } F_{X} /\left(q_{\infty} A\right) \\ C_{Y} & \text { Normal Force Coefficient, } F_{Y} /\left(q_{\infty} A\right) \\ P_{0} & \text { Freestream Total Pressure }\end{array}$

\section{INTRODUCTION}

The jet interaction flow field produced by a jet exhausting from the cone placed in a supersonic crossflow is a complex fluid dynamics problem with several real life applications. Figure 1 shows the schematic of the flow for lateral jet in supersonic flow. There are ample of the literature focused on the lateral jet. AGARD (1993) gives a detailed review of the field. The applications of the jet interaction phenomenon can be found from the very low-speed regimes of a chimney plume in a crossflow to the very high-speed hypersonic missile control systems. The basic problem of a gas injected into a crossflow has several variations due to different applications. Examples of these variables are the inclination of the injector, the jet flow conditions (subsonic, choked, supersonic), and on the freestream conditions (subsonic, supersonic, laminar, turbulent)

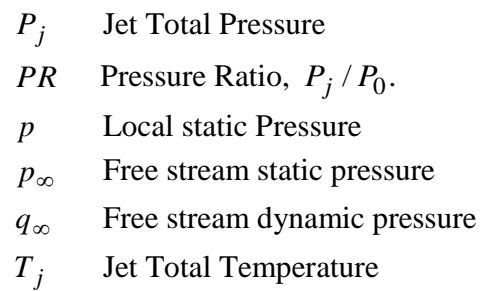

and inclination. Apart from these there are other variables such as the chemical composition of the gases (single or multiple-phase, non-reacting mixture, reacting mixture etc.) that make the computational effort even more demanding.

In the present study, we assumed a perfect gas is injected laterally from a cone through a sonic circular jet into a perfect gas supersonic oncoming flow and evaluate the effect of the parameters such as pressure ratio, and freestream flow inclination. This arrangement is representative of a reaction control systems installed on the hypersonic vehicles such as experimental aircraft (e.g. North American X-15), reentry vehicles (e.g. Space Shuttle, any future re-usable launch system, ICBMs) and missiles. An analogous approach replacing the jet thrusters with the pin protuberance installed on the outer surface of the vehicle were used for the 
directional control in a recent numerical study performed by Zahir et al. (2009). The study showed that the pin protuberance installed on the outer surface of a hypersonic vehicle, causes an increase in net force through altering pressure distribution in its near forward and aft vicinity, with subsequent development of an interaction aerodynamic force with usable pitching moment. However, thrusters or pin protuberance can also be used as directional control units when they are inserted in the exhaust nozzle of rocket-powered vehicles. The study performed by Walker et al. (1976) provides the baseline and more recently the numerical study of Ajmal et al. (2006) showed that a secondary jet is introduced into the primary exhaust flow of the nozzle. The jet interaction of the secondary jet with the primary flow depends upon the location where the secondary flow is injected into the primary flow of nozzle. The net result is the asymmetric thrust that causing a force normal to the flight direction of the vehicle. This normal force is usually larger than the thrust of the secondary jet.

The jet thrusters are usually preferred over the pin protuberance mainly due to the large force produced by the jet thruster and the fast reaction time. These thruster are typically used to intercept the high speed moving object in a low density atmosphere because of the fact that at this low density atmosphere, the conventional control system becomes ineffective due to very low dynamic pressure. This very low density atmosphere means that freestream flow conditions are on the edge of the boundary where the flow assumption may be regarded as a "continuum". In this study, continuous flow is assumed to exist for all the conditions examined. The normal injection is preferred over the inclined injection because it maximizes the lateral force produced by the thrust of the jet. There are two main sources that contribute to the production of the lateral force when the jet is injected in the crossflow. The first contribution comes from the thrust produced by the jet usually referred as the momentum thrust with the pressure correction. The second contribution comes from the complex interaction of the jet with the crossflow. The jet injected laterally into the oncoming flow acts as an obstruction to the primary flow and, as such, produces a shock wave in the primary flow as depicted in Fig. 1. This shock wave is referred to as the "jet bow shock". The jet bow shock produces an adverse pressure gradient that causes the boundary layer on the wall to separate ahead of the injector. Spaid et al. (1966) conducted experiment to study the secondary injection from a flat plate into a supersonic flow and showed that the high pressure ahead of the injector is the main source of the useful lateral force produced by the crossflow jet interaction phenomenon. This lateral force produced due to the crossflow jet interaction confirms that, a jet operating with a crossflow will produce a larger force than if it was exhausting into a quiescent medium. Meanwhile, there is also a suction region in the aft of the injector. In this suction region although pressure is not significantly lower than freestream pressure, it acts over a large area behind the injector, thus creating a strong negative force. This lowpressure region affects both the forces and moments produced by the jet interaction in the crossflow. The high pressure region ahead of the injector and the suction in the aft is mainly dependent on the pressure ratio $P R$. The second and in many aspects most important effect is the pitching moment that is generated with the high-pressure region ahead of the injector. This causes a nose-down moment about the injector.

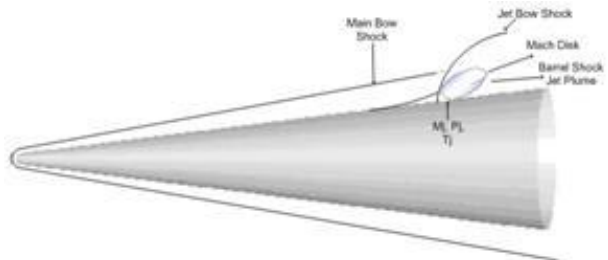

Fig. 1. Schematic of the flow structure for the jet exhausting in cross flow.

Viti et al. (2009) conducted the Numerical simulations of the 3D jet interaction flow field produced by a sonic circular jet exhausting normally into a turbulent supersonic cross flow over a flat plate were performed to study the time-averaged flow features that characterize this fluid-dynamic problem. The numerical computations made possible a detailed analysis of the prominent features that dominate the flow field. Zahir $e t$ al. (2009) conducted series of numerical simulations in order to evaluate the use used the Pin-Protuberances concept installed on a blunted cone body to provide possible directional control in supersonic flows.

In the present study the Pin-Protuberances proposed by Zahir et al. (2009) are replaced here with the jet on the basis of the study performed by Viti et al. (2009) with the jet issuing from the cone in cross flow at various pressure ratios to investigate the effectiveness of the jet by quantifying the useful pitching moment generated. Most of the flow features as mentioned Viti et al. (2009) for the jet issuing from flat plate are confirmed here when the jet issues from the cone in the crossflow. Apart from that the present study is also aimed to quantify the effectiveness of the jet in the form of useful pitching moment for directional control at varying pressure ratios and angle of attack. Further, a mathematical model is proposed for the forces and moments variation with pressure ratio, $P R$ and angle of $\operatorname{attack} \alpha$.

\section{GOVERNING EQUATIONS AND NUMERICAL METHODS}

The Reynolds averaged governing equations for an unsteady compressible turbulent flow is given by

$$
\frac{d}{d t} \int_{V(\vec{r}, t)} \vec{Q} d V+\oint_{\Omega(\vec{r}, t)}\left(\vec{F}_{c}-\vec{F}_{d}\right) \bullet \hat{n} d \Omega=\int_{V(\vec{r}, t)} \vec{S} d V
$$

where $V$ is control volume, $\Omega$ is the control surface. The first term in Eq. (1) is the time variation of $\vec{Q}$ in volume $V$, the second term is flux of $\vec{Q}$ through surface $\Omega$ and the right hand side term is the production of $\vec{Q}$ in volume $\mathrm{V}$. The vector $\vec{Q}$ contains the conservative variables:

$\vec{Q}=\left\{\rho, \rho u, \rho v, \rho w, \rho e_{0}, \rho k, \rho \omega\right\}^{T}$ 
where $\rho$ is the density, $u, v$ and $w$ are three Cartesian velocity components, and $e_{0}$ is the total energy of the flow.

$e_{0}=e+\frac{1}{2}\left(u^{2}+v^{2}+w^{2}\right)$

The flux vectors in Eq. (1) comprise the inviscid convective fluxes $\vec{F}_{c}$ and diffusive fluxes $\vec{F}_{d}$. For the convective fluxes, we include the pressure term and are given in Eq. (4).

$\vec{F}_{c}=\left\{\begin{array}{ccc}\rho u & \rho v & \rho w \\ \rho u^{2}+p & \rho u v & \rho u w \\ \rho v u & \rho v^{2}+p & \rho v w \\ \rho w u & \rho w v & \rho w^{2}+p \\ \rho e_{0} u+p u & \rho e_{0} v+p v & \rho e_{0} w+p w \\ \rho u k & \rho v k & \rho w k \\ \rho u \omega & \rho v \omega & \rho w \omega\end{array}\right\}$

For the diffusive fluxes, we have

$$
\vec{F}_{d}=\left\{\begin{array}{ccc}
0 & 0 & 0 \\
\tau_{x x} & \tau_{x y} & \tau_{x z} \\
\tau_{y x} & \tau_{y y} & \tau_{y z} \\
\tau_{z x} & \tau_{z y} & \tau_{z z} \\
u \tau_{x x}+v \tau_{x y}+w \tau_{x z}-q_{x} & u \tau_{y x}+v \tau_{y y}+w \tau_{y z}-q_{y} & u \tau_{z x}+v \tau_{z y}+w \tau_{z z}-q_{z} \\
\mu^{*} \frac{\partial \kappa}{\partial x} & \mu^{\frac{\partial}{\partial}} \frac{\partial \kappa}{\partial y} & \mu^{*} \frac{\partial \kappa}{\partial z} \\
\mu^{*} \frac{\partial \omega}{\partial x} & \mu^{*} \frac{\partial \omega}{\partial y} & \mu^{*} \frac{\partial \omega}{\partial z}
\end{array}\right\}
$$

The source term $\vec{S}$ is defined as

$$
\vec{S}=\left\{\begin{array}{c}
0 \\
0 \\
0 \\
0 \\
0 \\
\beta-\rho \omega \\
\left(C_{\varepsilon 1} \beta-C_{\varepsilon 2} \rho \omega\right) \frac{\omega}{k}
\end{array}\right\}
$$

The laminar viscosity $\mu_{l}$ is obtained by Sutherland's law and by Wilcox (1998) k- $\omega$ turbulence model is employed.

The shear stresses and the heat fluxes follow the customary Newton and Fourier law and are defined as

$$
\begin{aligned}
& \tau_{x x}=2 \mu^{*} \frac{\partial u}{\partial x}-\frac{2}{3} \mu^{*}\left(\frac{\partial u}{\partial x}+\frac{\partial v}{\partial y}+\frac{\partial w}{\partial z}\right)-\frac{2}{3} \rho \kappa \\
& \tau_{z z}=2 \mu^{*} \frac{\partial w}{\partial z}-\frac{2}{3} \mu^{*}\left(\frac{\partial u}{\partial x}+\frac{\partial v}{\partial y}+\frac{\partial w}{\partial z}\right)-\frac{2}{3} \rho \kappa \\
& \tau_{x y}=\tau_{y x}=\mu^{*}\left(\frac{\partial u}{\partial y}+\frac{\partial v}{\partial x}\right) \\
& \tau_{y y}=2 \mu^{*} \frac{\partial v}{\partial y}-\frac{2}{3} \mu^{*}\left(\frac{\partial u}{\partial x}+\frac{\partial v}{\partial y}+\frac{\partial w}{\partial z}\right)-\frac{2}{3} \rho \kappa
\end{aligned}
$$

$$
\begin{aligned}
& \tau_{y z}=\tau_{z y}=\mu^{*}\left(\frac{\partial v}{\partial z}+\frac{\partial w}{\partial y}\right) \\
& \tau_{x z}=\tau_{z x}=\mu^{*}\left(\frac{\partial w}{\partial x}+\frac{\partial u}{\partial z}\right) \\
& q_{z}=-\left(\kappa_{L}+\kappa_{T}\right) \frac{\partial T}{\partial z} \\
& q_{y}=-\left(\kappa_{L}+\kappa_{T}\right) \frac{\partial T}{\partial y} \\
& q_{x}=-\left(\kappa_{L}+\kappa_{T}\right) \frac{\partial T}{\partial x}
\end{aligned}
$$

Here $k_{L}$ is the laminar thermal conductivity and $k_{T}$ is the turbulent thermal conductivity.

The production $\beta$ used in Eq. (6) is defined as

$$
\beta=\left[\mu_{t}\left(\frac{\partial u_{i}}{\partial x_{j}}+\frac{\partial u_{j}}{\partial x_{i}}-\frac{2}{3} \delta_{i j} \frac{\partial u_{k}}{\partial x_{k}}\right)-\frac{2}{3} \delta_{i j} \rho \kappa\right] \frac{\partial u_{i}}{\partial x_{j}}
$$

where $\delta_{i j}$ is Kronecker delta function. The subscripts $i, j=1,2,3$ are in $x, y$ and $z$-direction respectively.

After representing the integral Eq. (1) into the ordinary differential equation and eventually transforming it to the algebraic equation for a mesh that is comprised of discrete volumes and area, the above equation is transformed to an implicit formulation at time level $\mathrm{n}+1$,

$\frac{\partial(\vec{Q} V)}{\partial t}+\sum_{f=1}^{N_{\text {face }}}\left(\vec{F}_{C}-\vec{F}_{D}\right)_{f}^{n+1} \hat{n}_{f}^{n+1} \Delta A_{f}^{n+1}=\vec{S}^{n+1} \Delta V^{n+1}$

$\hat{n}=$ Cell face normal

$\Delta A_{f}=$ The cell face area

$\Delta V=$ The cell volume

The flux and source vectors must be linearized to estimate the fluxes and sources at $\mathrm{n}+1$. This gives (temporarily dropping the subscripts),

$\vec{F}_{f}^{n+1}=\vec{F}_{f}^{n}+\left.\frac{\partial \vec{F}_{f}}{\partial \vec{Q}}\right|_{f} \Delta \vec{Q}$

$\vec{S}_{\text {cell }}^{n+1}=\vec{S}_{\text {cell }}^{n}+\left.\frac{\partial \vec{S}}{\partial \vec{Q}}\right|_{\text {cell }} \Delta \vec{Q}$

$\delta \vec{Q} V=V^{n+1} \Delta \vec{Q}^{n}-\vec{Q}^{n} \delta V^{n}$

where $\frac{\partial \vec{F}_{f}}{\partial \vec{Q}}$ and $\frac{\partial \vec{S}}{\partial \vec{Q}}$ are the flux Jacobian and source Jacobian, respectively.

Rearranging the terms in Eq. (8) gives,

$$
\begin{aligned}
& \frac{\Delta \vec{Q}}{\Delta t} \Delta V^{n+1}+\sum_{f=1}^{N f a c e}\left[\left(\left.\frac{\partial \vec{F}_{C}}{\partial \vec{Q}}\right|_{f} ^{n}-\left.\frac{\partial \vec{F}_{D}}{\partial \vec{Q}}\right|_{f} ^{n}\right) \Delta \vec{Q} \bullet \hat{n}_{f} \Delta A_{f}\right] \\
& -\left(\Delta V \frac{\partial \vec{S}^{n}}{\partial \vec{Q}}\right) \Delta \vec{Q}=\vec{S} \Delta V^{n+1}-\sum_{f=1}^{N \text { fface }} \vec{F}_{f}^{n} \bullet \hat{n}_{f} \Delta A_{f}+\vec{Q}^{n} \delta V^{n}
\end{aligned}
$$


For non moving grids, $\delta V^{n}=0, \Delta V^{n+1}=V^{n}$ and $\Delta A^{n+1}=\Delta A^{n}$.

The present formulation uses the upwind scheme for convective fluxes. In particular, we use here the Van Leer's scheme based on flux vector splitting. The use of limiters makes this scheme 2nd order accurate. In particular the Van Leer limiters are used. The detail of the Van Leer scheme and use of limiter can be found in Hirsch (2007). A central difference scheme is used to discretize the diffusive terms. The block diagonal iterative method and a Jacobi-point iterative method are used for the time integration. The presented code PAK$3 \mathrm{D}$ has been validated in our previous studies such as by Zahir et al. (2009) in similar studies performed where the Pin-Protuberances instead of a jet for directional control and more recently by Shah and $\mathrm{Lu}$ (2010) where jet exhaust in the counterflow direction has been studied extensively.

\section{COMPUTATIONAL DOMAIN AND BOUNDARY CONDITIONS}

The freestream conditions for the incoming flow are shown in Table 1 . The jet conditions for the different pressure ratios are mentioned in Table 2. The freestream static temperature remains fixed at $73 \mathrm{~K}$. The jet total pressure is varied to account for the relative mass flow variation. The sonic jet with static temperature of $261 \mathrm{~K}$ was kept constant for all the cases.

Table 1 Freestream conditions for the incoming flow

\begin{tabular}{|c|c|c|}
\hline $\begin{array}{c}\text { Free Stream } \\
\text { Mach Number }\end{array}$ & $p_{\infty}[\mathrm{N} / \mathrm{m} 2]$ & $\rho_{\infty}[\mathrm{Kg} / \mathrm{m} 3]$ \\
\hline 4.0 & 7100 & 0.35190 \\
\hline
\end{tabular}

Table 2 Jet Conditions used and flow mode achieved for freestream flow Mach number 4.0

\begin{tabular}{|l|l|l|}
\hline Jet Mach Number & $p_{\infty}[\mathrm{N} / \mathrm{m} 2]$ & $\rho_{\infty}[\mathrm{Kg} / \mathrm{m} 3]$ \\
\hline 1.0 & 7100 & 0.35190 \\
\hline
\end{tabular}
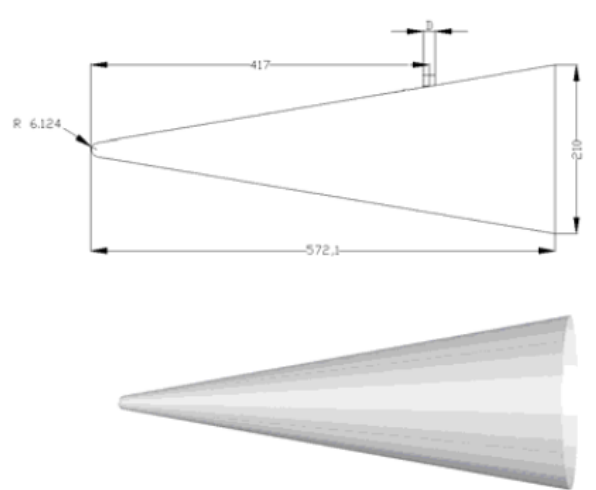

Fig. 2. Generic Cone Geometry with location of Jet.

The model selected here was a blunted cone cylinder configuration having a blunted cone with a half-cone angle of $10.4^{\circ}$, with and its base diameter is $0.21 \mathrm{~m}$ and the nose radius is $0.01 \mathrm{~m}$. Geometrical features are described in Fig. 2. The jet centre line is at $0.417 \mathrm{~m}$ form the nose tip, and the diameter (D) of the jet is same as studied by Zahir et al. (2009), which is $0.0141 \mathrm{~m}$.

From the simple H-type topology, the grid evolved into a more complex combination of $\mathrm{H}$-type and C-type grids with better cell clustering in the injector and jet plume regions (see Fig. 3). A C-type grid can wrap around the injector, and any cell clustering will not be propagated far away from the injector but will stay concentrated around the jet. In fact, the complicated flow physics and the steep pressure gradients found in this region require a finely spaced grid to be accurately resolved. A further and final grid adaptation step produced an $\mathrm{H}$-type and C-type grid combination with contoured zonal boundaries. The contoured zonal boundaries improved the cell distribution all through the C-grid surrounding the jet thus improving the grid quality.

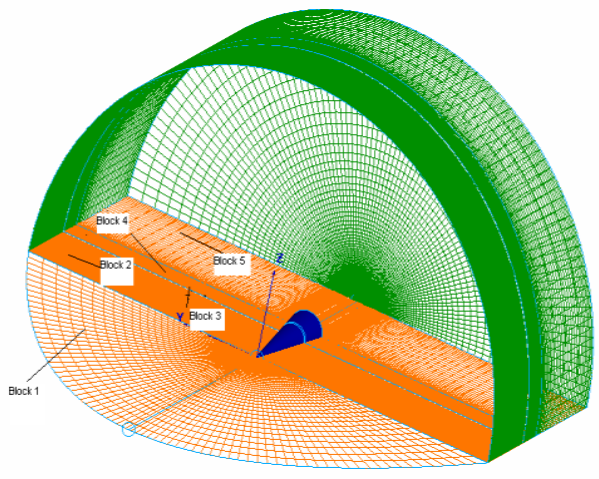

Fig. 3. Overview of the computational grid

Three different mesh sizes with 3D-plnaer symmetric case were used that comprises the 5 blocks. The grid size ranged from 1.0 million cells to 1.5 million cells. After the grid independence study a mesh size of 1.5 million was selected for detailed flow analysis. The grid size was dictated by the need to find a balance between the grid refinement and the time to converge a solution to a steady state. On average, it would take 2090 total CPU hours using SGI Origin CPUs to achieve a converged turbulent solution using a 1.5 million-cell grid. The computational domain covered only half of the cone, since it was assumed that a longitudinal plane of symmetry passing through the primary jet centerline existed. The origin of the right-handed coordinate axes used in all of the jet interaction simulations is located at the center of the injector. The positive $\mathrm{X}$-coordinate is downstream; the positive Y-coordinate is away from the top surface of the cone. The adequacy of the normal spacing was assessed by calculating the $y^{+}$for the cells on the cone surface ahead of the separation region. All the cells adjacent to the solid surface were below $y^{+}$of 1.0. This spacing close to the wall is a widely accepted value for accurate predictions using the $k-\omega$ turbulent model in wall-bounded turbulent flows. The injector 
was simulated by cells on the surface of the cone with imposed pressure and velocity equal to the jet total conditions. Due to the plane of symmetry of the problem only half of the injector was simulated.

The typical computational domain for the flow over a cone with normal injection consisted of a block with six-sided domains referred as I1, ILAST, J1, JLAST, $\mathrm{K} 1$ and KLAST. The lower plane J1, i.e. the plane defined by $\mathrm{y} / \mathrm{d}=0.00$, corresponds to the solid surface of the cone and the jet. The upper plane JLAST corresponds to inflow boundary. The plane I1, K1 and KLAST corresponds to the plane of symmetry $(\mathrm{z} / \mathrm{d}=0.00)$ and ILAST corresponds to the exit plane. The plane defining the entry boundary conditions are set at freestream conditions. The following is a general description of the applied boundary conditions.

\section{RESUltS AND DisCUSSION}

\subsection{Vortical Structure of Jet Interaction Flowfield}

The jet interaction flowfield arising from the jet injected into a supersonic crossflow is a very complicated flowfield. There are strong jet bow shocks along with separated flows, expansion fans, reflected shocks, a Mach disk, viscous interaction and a complex system of trailing vortices. The analysis presented here is based on the numerical results obtained from the simulation of the freestream Mach 4.0 with varying pressure ratios of the jet as mentioned in Table 2 . Figure 4 shows the axial $\mathrm{Cp}$ distribution in vicinity of the lateral jet in the meridian plane with jet injection at pressure ratio $P R=3.52$. The flow structure and the various regions identified on the plot shows very complex flow structure on the centerline as mentioned by Viti et al. (2009).

Following the flow along its path from right to left, the first flow region referred in Fig. 4, as region 1 is encountered that is produced by the undisturbed freestream flow over the cone where the Mach number on the solid surface of the cone is zero, and it gradually increases to the freestream conditions as we move away from the solid wall. Region 2 shows the steep peak in $\mathrm{Cp}$ plot followed by a pleatue (Region 3 ) that are due to the flow separation caused by the shock-boundary layer interaction and is referred to as the $\lambda$ shock. The strong pressure gradient caused by the jet bow shock propagates upstream through the boundary layer. The pleatue in the Cp plot referred as the region 3 is the core of the horseshoe vortex formation and is shed sideways from the symmetry plane highlighted by the streamlines are also seen in Fig. 5.

The boundary between regions 3 and 4 , where there is a decrease in the pressure along the center line defines stagnation location of the two counter-rotating vortices both of which are visible through the streamlines. As noted by Viti et al. (2009), the peak in region 5 defines the attachment line in the two counter rotating vortices. The peak in $\mathrm{Cp}$ plot of region 6 and 7 are also consistent as being the highest values in front of the injector. As mentioned by Viti et al. (2009), "the two counter-rotating vortices in the separation region create one strong vortical structure that is the horseshoe vortex." As pressure ratio increases, the horseshoe vortex moves away from the symmetry plane. The second counter rotating vortex form a single coherent structure at lower pressure ratios, as is evident in the cross plane of Fig. 5a through $6 \mathrm{c}$.

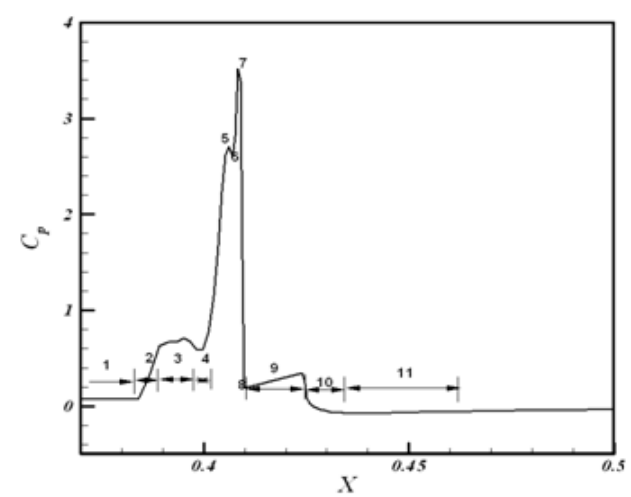

Fig. 4. Axial Cp distribution in vicinity of the lateral jet in the symmetric plane with jet injection at pressure ratio $P R=3.52$

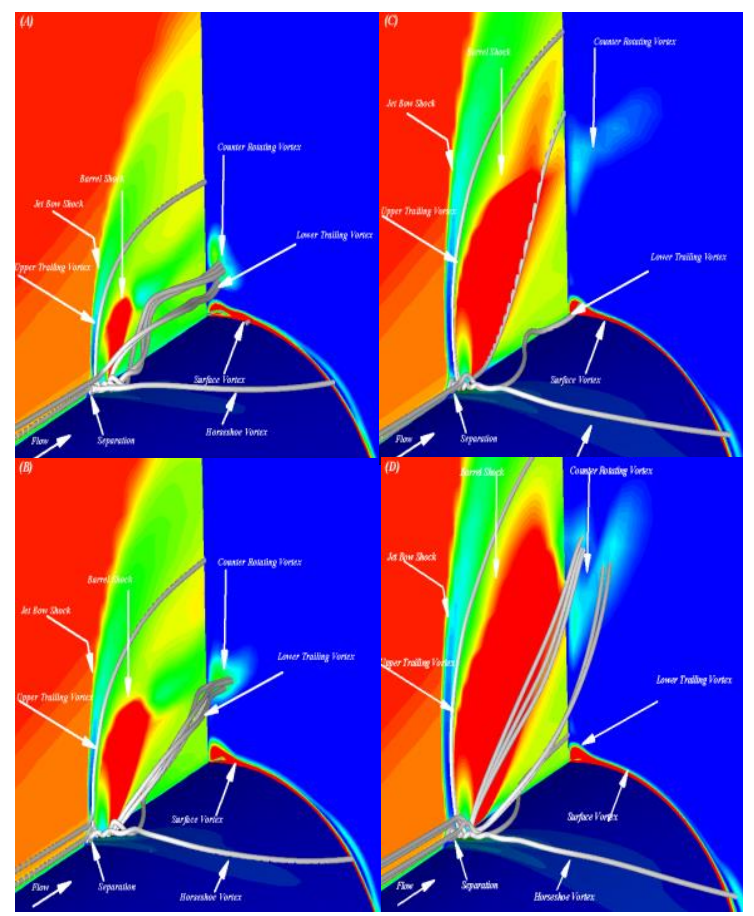

Fig. 5. Isometric view of the flow around the injector with streamlines highlighting the main vortical structures. Mach number contours on symmetry plane, $\mathrm{Cp}$ contours on surface of flat plate, vorticity magnitude contours on cross plane, (a) $\mathrm{PR}=0.66$; (b) $\mathrm{PR}=1.32$; (c) $\mathrm{PR}=2.65$; (d) $\mathrm{PR}=3.52$.

At higher pressure ratio, $P R=3.53$, the second counter rotating vortex does not generate one single coherent structure but rather it generates several smaller vortical structures that trail downstream and around the barrel shock as shown in the cross plane of the Fig. 5d. The second counter rotating vortex has the more complicated trace and the fluid is dispersed in various directions. Some of the fluid follows the leading edge 
of the barrel shock away from the solid surface and it is visible in Fig. 5a through 5d. The rest of the fluid of the second counter-rotating vortex is moved downstream sideways. The surface trailing vortex close to the surface of the cone is also evident.

\subsection{Shock Structure and Pressure Distribution}

Figure 6 shows the axial $\mathrm{Cp}$ distribution in vicinity of the lateral jet in the meridian plane at various injection pressure ratios. It is evident that the surface pressure and the separation region increase with the increase in injection pressure ratio. The overall effect of the injection pressure ratio can be seen in the integrated normal force and pitching moments.

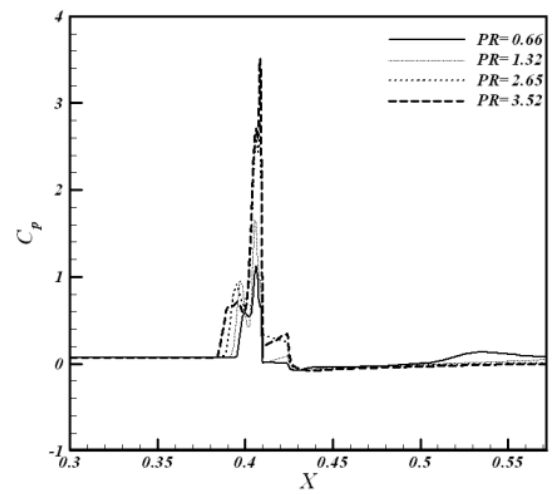

Fig. 6. Axial $\mathrm{Cp}$ distribution in vicinity of the lateral jet in the symmetric plane with jet injection at various pressure ratios.

Figure 7 shows the Mach number contours along the plane of symmetry at various pressure ratios. Figure 7 also shows the formation well defined separation induced shock referred as a $\lambda$ shock. This shock is formed just ahead of the jet barrel shock and, it appears as the Greek letter " $\lambda$ ". In reality the " $\lambda$-shaped" shock is formed as a result of mergence of the separation-induced shock and the part of the jet bow shock. The jet bow shock is formed in front of the barrel shock. The jet bow shock is the result of the obstruction as the "barrel shock" formed acts as a solid boundary for the jet bow shock. When the bow shock moves away from the plane of symmetry and from the cone surface it becomes a curved shock around the jet plume. The jet bow shock pattern is very similar to that of the bow shock generated by a blunt body in a threedimensional flow. The similarity of the "jet bow shock" and "main bow shock" is consistent with the findings of various researches to correlate the two flowfields identified by the experimental work of Spaid et al. (1996) and Strike (1963) and later by Viti et al. (2009) in his computational study. In comparison with the flow structure of jet exhausting in quiescent flow that has been extensively studied by Woodmansee et al. (1999) and later by McDaniel et al. (2002).

Figure 7 shows the typical flow structures for a sonic jet exhausting into the supersonic crossflow. Figure $7 \mathrm{a}$ through $7 \mathrm{~d}$ shows the Mach number contours at various pressure ratios along the plane of symmetry. This barrel shock and flow structure is similar to that of a typical under-expanded jet exhausting in a quiescent flow.

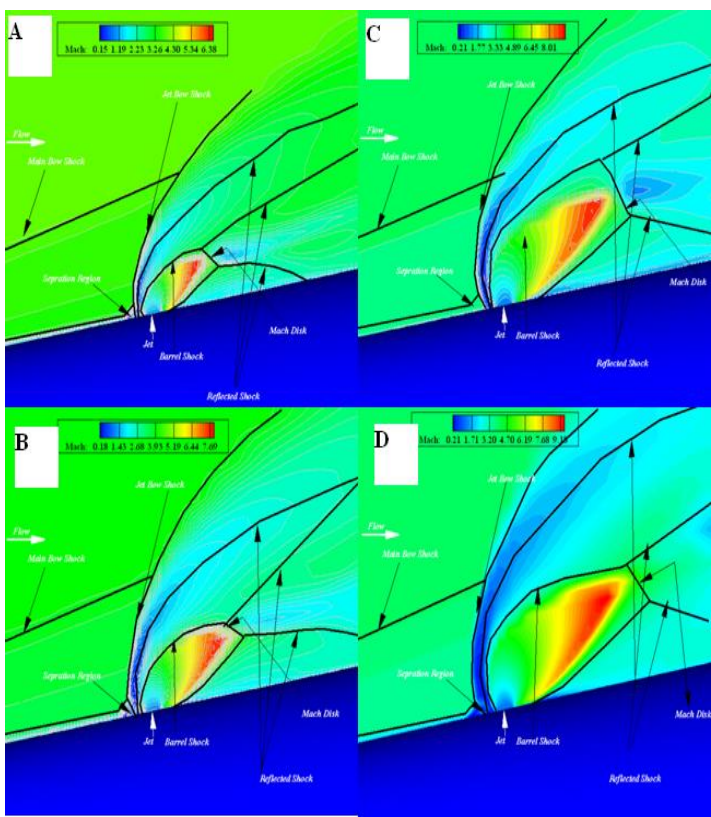

Fig. 7. Isometric view of the shock structure with Mach number contours on symmetry plane at various pressure ratios (a) $\mathrm{PR}=0.66$; (b) $\mathrm{PR}=1.32$; (c) $\mathrm{PR}=2.65$; (d)

$\mathrm{PR}=3.52$.

However, because of the crossflow in the jet interaction flowfield, the back pressure is not constant around the expanding jet. The back pressure is much higher on the windward side of the plume than on the leeward side. This higher pressure on the windward side of the plume forces the plume to trail downstream and hence the plume looses its symmetry. Moreover, the windward side of the barrel shock has less resemblance to the under-expanded jet than the leeward side. This is due to the fact that there is a strong bow shock in front of the windward side of barrel shock.

For a short distance, the leeward side of the barrel shock is attached to the solid surface of the cone. With the increase in pressure ratio, this attachment distances decreases. As the pressure ratio increases, the volume of fluid inside the barrel shock increases. There is a formation of the reflected shock emerging from the triple point and is clearly identified in Fig. 7. This reflected shock impinges on the cone only at the lowest pressure ratio of pressure ratio as shown in Fig. 7a.

This can also be verified by the Cp plot shown in Fig. 6 . There is a rise in pressure at the cone surface around $X=0.54$ for $P R=0.66$ only. With the increase in pressure ratio, the reflected shock never impinges on the cone surface as shown in Fig. $7 \mathrm{~b}$ through $8 \mathrm{~d}$ and is confirmed in the Cp plot of Fig. 6.

\subsection{Forces and Moments Variation with Pressure Ratio and Angle of Attack}

The jet interaction phenomenon gave rise to a high pressure region ahead of the injector and a low pressure region in the aft of the injector at a typical pressure ratio. At each pressure ratio $P R$, the study is further extended to determine the effect of angle of attack $\alpha$. At a pressure ratio $P R=3.52$, Figure 8 shows the 
pressure distribution in the form of normalized $\mathrm{Cp}$ for various angles of attack $\alpha$. Figure 8 a through to $8 \mathrm{c}$ shows the $\mathrm{Cp}$ distribution when the angle of attack is negative.

At highest negative angle of attack $\alpha=-10$, there is a strong interference between the main bow shock and the jet and as a result the pressure in the front of injector is also highest as shown in Fig. 8a.With the decrease in angle of attack $\alpha$, this strong interference between the main bow shock and the jet decreases and can be seen by following the Figs. 8 a through to 9f. At an angle of attack $\alpha=10$, with the jet being installed in the leeward side, the interference effects between the main bow shock and the jet are minimal and hence the pressure in front of the injector decreases.

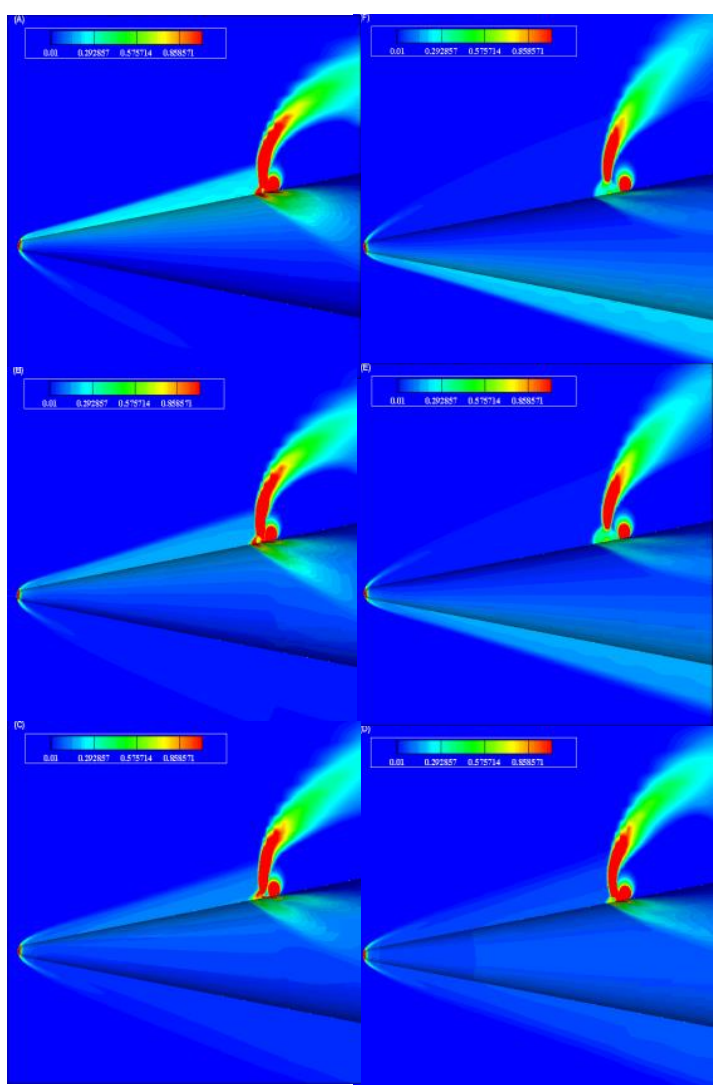

Fig. 8. Cp distribution showing the effect of angle of attack on the cone and symmetry plane (a) $\alpha=-10$,(b) $\alpha=-6$,(c) $\alpha=-4$,(d) $\alpha=0$,(e) $\alpha=6$,(f) $\alpha=10, \mathrm{PR}=3.52$,

$$
M_{\infty}=4.0
$$

This interference between the main bow shock and the jet with variation in angle of attack $\alpha$ is important and must be quantified. The overall effect of the main bow shock jet interaction is quantified when the pressure on the cone surface is integrated. The integration of the pressure on the cone surface results in axial force coefficient $C_{X}$, normal force coefficient $C_{Y}$, and the resulting pitching moment coefficient $C_{M}$. For angle of attack $\alpha=0$, there is a non-linear rise in axial force coefficient $C_{X}$ with pressure ratio $P R$ and is shown in Fig. 9. The normal force coefficient $C_{Y}$ and the pitching moment coefficient $C_{M}$ vary linearly with pressure ratio $P R$ and are shown in Figs. 10 and 11 respectively.

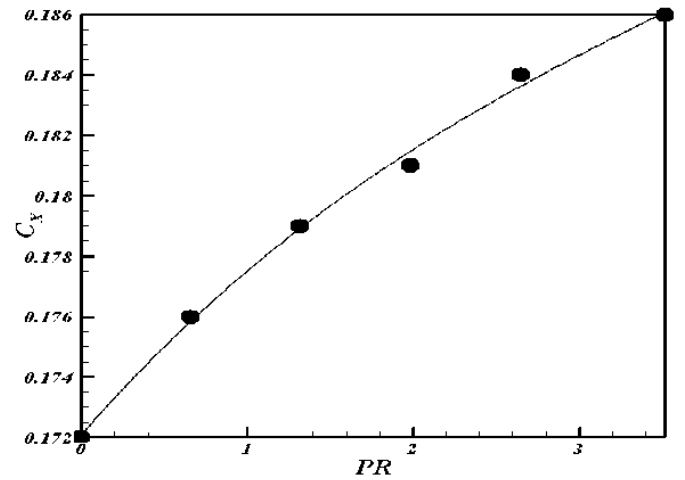

Fig. 9. Axial force coefficient $C_{X}$ variation with pressure ratios $\mathrm{PR}$.

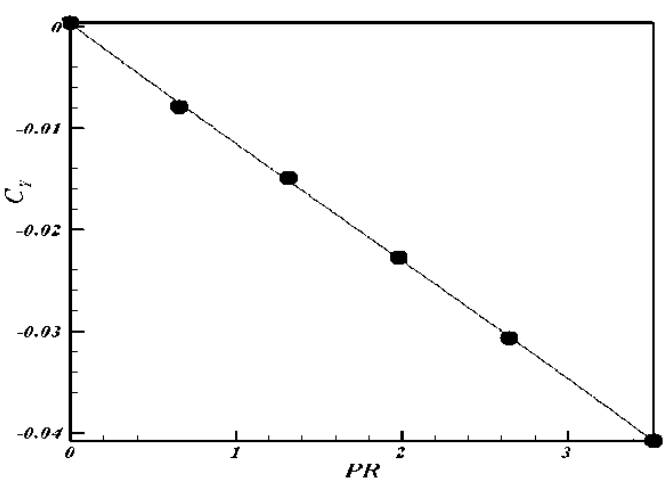

Fig. 10. Normal force coefficient $C_{Y}$ variation with pressure ratios $\mathrm{PR}$.

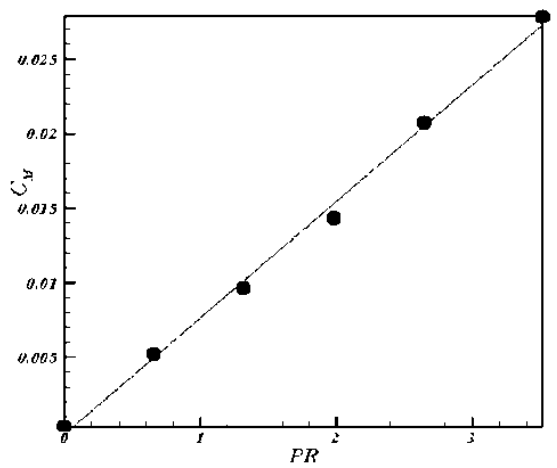

Fig. 11. Pitching moment coefficient $C_{M}$ variation with pressure ratios $\mathrm{PR}$

Figure 12 shows the rise in the axial force coefficient $C_{X}$ with angle of attack $\alpha$ at various pressure ratios $P R$. The axial force coefficient $C_{X}$ follows conventional parabolic behavior with angle of attack $\alpha$. However for $\alpha<0$, due to strong interference between the main bow shock and the jet, the axial force is slightly higher then for $\alpha>0$. Similar interference effects between the main bow shock and the jet can also be seen for normal force coefficient $C_{Y}$ and the pitching moment coefficient $C_{M}$. 


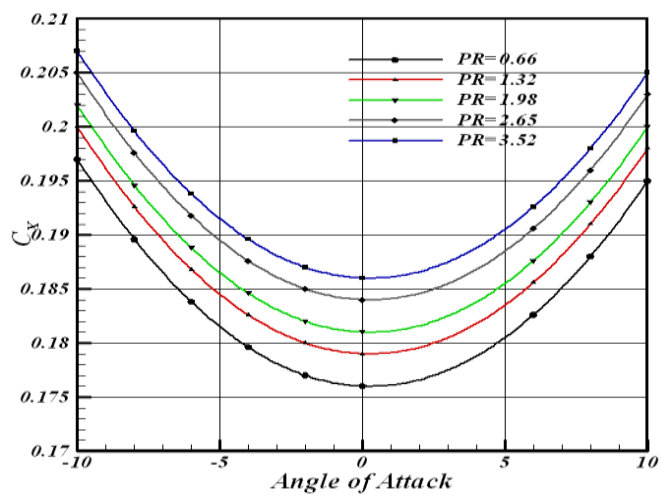

Fig. 12. Axial force coefficient $C_{X}$ variation with the angle of attack at various pressure ratios PR.

Figures 13 and 14 shows the normal force coefficient $C_{Y}$ and the pitching moment coefficient $C_{M}$ variation with angle of attack $\alpha$ for various pressure ratios, $P R$ respectively. Due to the strong interference between the main bow shock and the jet at negative angle of attack $\alpha$, added effectiveness gives rise to the amplification of pitching moment $C_{M}$

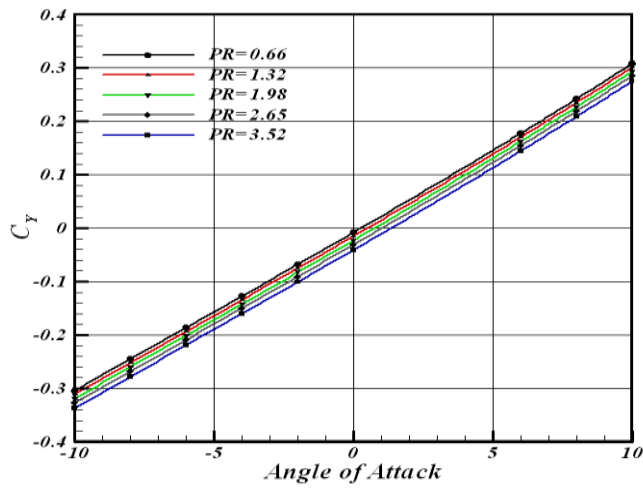

Fig. 13. Normal force coefficient $C_{Y}$ variation with the angle of attack at various pressure ratios $\mathrm{PR}$

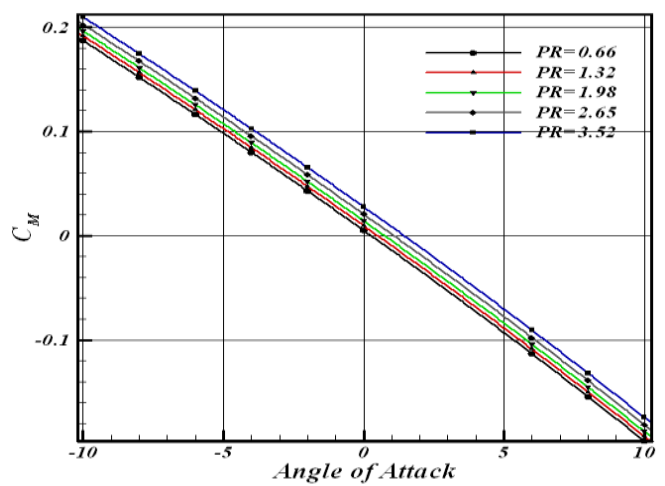

Fig. 14. Pitching moment coefficient $C_{M}$ variation with the angle of attack at various pressure ratios PR.

The data presented in Figs. 12, 13 and 14 can be expressed in the mathematical form representing the axial force coefficient, $C_{X}$, normal force coefficient,
$C_{Y}$ and pitching moment coefficient, $C_{M}$ and is given as in Eq. (10) through (12).

$$
\begin{aligned}
& C_{X}=0.1773+0.000791678 P R^{2}+0.0002 \alpha^{2}-0.0001 \alpha \\
& C_{Y}=-0.01193 P R+0.0305087 \alpha+0.0001 \alpha^{2} \\
& C_{M}=0.00789 P R-0.019209 z \alpha-0.0001 \alpha^{2}
\end{aligned}
$$

The squared value of the correlation coefficient, $r$ for the linear regression given by Eq. (10) through (12) is 0.988 .

\section{CONClusion}

It has been concluded that CFD simulations showed that a crossflow jet interaction with the oncoming freestream flow over a blunted cone, causes an increase in net force through altering pressure distribution in its near forward and aft vicinity of the jet, with subsequent development of an interaction aerodynamic force with usable pitching moment, which possibly had a further application for flight control under supersonic flight conditions of Mach 4 under varying incidence angle combinations. Flow interaction resulting in generation of a pitching moment was confirmed when the Mach number remained fixed at Mach 4 and a corresponding increase in the angle of attack was made, simultaneously with an increase of jet pressure ratio, $P R$ from 0.33 to 3.52. Computed static aerodynamic coefficients with $P R$ were presented to demonstrate this interaction effect and further pressure and Mach contours with streamline patterns gave a qualitative insight. All computed static aerodynamic coefficients and pressure distribution using CFD analysis is with an accuracy of $\pm 5 \%$ for the supersonic Mach investigated.

\section{REFERENCES}

Behrouzi, P. and J.J. McGuirk (1993, April). Experimental Data for CFD Evaluation of Jets with or without Cross Flow Effects for ASTOVL Flow Application. AGARD Conference Proceedings 534, Computational and Experimental Assessment of Jets in Cross flow, Winchester, UK, 8.1-8.11.

Ajmal, B., S. Zahir and A. Ahmed (2006, September). Numerical Study of Effects of injection pressure and location of secondary injection inside a low thrust Satellites nozzle. 25th Congress of International Council of the Aeronautical Sciences, Paper ICAS 2006-P2.10, Hamburg, Germany.

Shah, S.B.H. and X.Y. Lu (2010). Computational Study for Drag reduction at various freestream flows using a counterflow jet from hemisphere cylinder. 
S.B.H. Shah et al. / JAFM, Vol. 5, No. 1, pp. 39-47, 2012.

Engineering Application of Computational Fluid Mechanics 4(1), 150-163.

Hirsch, C. (2007). Numerical Computations of Internal and External Flows. Vol. I and II., Second edition, John Wiley \& Sons, Ltd.

McDaniel, J., C. Glas, D. Staack, and C. Miller (2002). Experimental and computational comparison of an under-expanded jet flowfield. AIAA Paper No. 2002-0305.

Spaid, F.W., E.E. Zukoski and R. Rosen (1996). A study of secondary injection of gases into a supersonic flow. Jet Propulsion Laboratory, California Institute of Technology, Pasadena, CA, NACA Technical Report No. 32-834.

Strike, W.T. and C.J. Schueler (1963). Investigation of interference effects produced by lateral jets on surfaces in a supersonic stream. AIAA 63-184.

Viti, V., N. Reece and Schetz, J.A. (2009). Detailed flow physics of the supersonic jet interaction flow field. Physics of Fluids 21, 046101.

Walker, R.E., A.R. Stone, and M. Shandor (1963). Secondary gas injection in a conical rocket nozzle. I. Effect of Orifice diameter and molecular weight of injectant. AIAA Journal 1, 334-338.

Wilcox, D.C. (1998). Turbulence Modeling for CFD. ISBN 1-928729-10-X, 2nd Ed., DCW Industries, Inc.

Woodmansee, M.A. and J.C. Dutton (1999). Experimental measurements of pressure, temperature, and density in an under-expanded sonic jet flowfield. AIAA Paper No. 99-3600.

Shah, S.B.H., S. Zahir and X.Y. Lu (2009). Computational Aerodynamic Simulations for PinProtuberances in Supersonic Flow. EUCASS 2009292. 